\title{
Anxiety and Depression Associated with Anal Sexual Practices among HIV-Negative Men Who Have Sex with Men in Western China
}

\author{
Jiaxiu Liu ${ }^{1}$, Xiaoni Zhong ${ }^{1, *}$, Zhuo Lu ${ }^{1}$, Bin Peng ${ }^{1}$, Yan Zhang ${ }^{1}$, Hao Liang ${ }^{2}$, Jianghong Dai ${ }^{3}$, \\ Juying Zhang ${ }^{4}$ and Ailong Huang ${ }^{5}$ \\ 1 School of Public Health and Management, Chongqing Medical University, Chongqing 400016, China; \\ jiaxiu219@gmail.com (J.L.); lz18084084564@163.com (Z.L.); pengbin@cqmu.edu.cn (B.P.); \\ cqmudzy@163.com (Y.Z.) \\ 2 Department of Epidemiology and Medical Statistics, School of Public Health, Guangxi Medical University, \\ Nanning 520021, China; haolphd@163.com \\ 3 Department of Epidemiology and Health Statistics, School of Public Health, Xinjiang Medical University, \\ Xinjiang 830011, China; epi102@sina.com \\ 4 Department of Epidemiology and Medical Statistics, School of Public Health, Sichuan University, Chengdu \\ 610041, China; juying109@163.com \\ 5 Key Laboratory of Molecular Biology on Infectious Diseases, Ministry of Education, Chongqing Medical \\ University, Chongqing 400016, China; Ahuang1964@163.com \\ * Correspondence: zhongxiaoni@cqmu.edu.cn
}

Received: 25 November 2019; Accepted: 8 January 2020; Published: 10 January 2020

\begin{abstract}
This study aimed to explore the prevalence and influencing factors of anxiety and depression among human immunodeficiency virus negative (HIV-negative) men who have sex with men (MSM) based on anal sex roles, so as to provide a scientific basis for the management of mental health conditions. Data were obtained from the baseline in a cohort study with a two-year follow-up period in western China. The Self-Rating Anxiety Scale and Center for Epidemiological Studies Depression Scale were used to assess anxiety and depression symptoms, respectively. The prevalence of anxiety and depression in $1771 \mathrm{MSM}$ was $26.03 \%$ and $37.83 \%$. Among them, 182 anal sex role " 0 " MSM who only had receptive anal sex with men reported the highest prevalence of anxiety and depression $(31.32 \%$ and $46.15 \%), 467$ anal sex role "1" MSM who only had insertive anal sex with men $(22.27 \%$ and $32.76 \%$ ), and 1122 anal sex role " 0.5 " MSM who engaged equally in both insertive and receptive anal sex intercourse with men $(26.74 \%$ and $38.59 \%)$, respectively. Logistic regression analysis revealed that the influencing factors of anxiety include anal sex role " 0 " $\mid$ " 0.5 ", low educational level, female sexual partners, frequently searching partners on the Internet, sexually transmitted diseases (STD) diagnosed by doctors, and no HIV counseling. Anal sex role " 0 "|" 0.5 ", rural area, casual male partners, female partners, STD diagnosed by doctors, frequently searching partners on the Internet, no HIV counseling, no condom use, and daily alcohol use were found to be associated with depression. In conclusion, early identification and intervention of anxiety and depression symptoms in HIV-negative MSM should be carried out, especially for anal sex role " 0 " MSM. Furthermore, key intervention and psychological counseling should be taken into consideration for MSM with a low education level, high-risk behaviors, and high-risk perceptions.
\end{abstract}

Keywords: anxiety; depression; HIV-negative MSM; anal sex role; influencing factors; western China

\section{Introduction}

In China, HIV (human immunodeficiency virus) prevalence has declined in recent years, while men who have sex with men (MSM) have become the most important high-risk group for new HIV 
infection [1-3]. The Joint UN Programme on HIV/AIDS (acquired immunodeficiency syndrome) report [4] also pointed out that MSM are 19 times more likely to be infected with HIV than the general population, which also brings a lot of mental health problems. Moreover, MSM were found to be three times more likely to suffer from mental disorders, such as anxiety and depression, than heterosexuals [5].

Globally, the social acceptance of MSM is not high in most countries and regions [6,7], and there is widespread prejudice or even discrimination against MSM, which leads to a certain degree of mental health problems in MSM. In previous studies, the prevalence of anxiety in the general population was less than $10 \%$ [8], and that of depression was between $5 \%$ and $12 \%$ [9] while the prevalence of anxiety in MSM was much higher than that of the general population $[8,10,11]$. The prevalence of anxiety and depression in an MSM population in Canada [12] and the United States [13] ranged from $26.4 \%$ to $44 \%$ and $39.3 \%$ to $80 \%$, respectively, and in China [14-16], these figures were about $21.25 \%$ to $24.0 \%$ and $33.2 \%$ to $45.4 \%$, respectively, suggesting that mental health in MSM could not be ignored.

Compared with traditional heterosexual behavior, anal sex and oral sex are mainly sexual behaviors of MSM, especially anal sex behaviors, which are generally expressed as " 1 " and " 0 " for insertive and receptive anal sex, respectively $[17,18]$. As is well-known, because of the special tissue structure of the anus, the risk of sexually transmitted diseases (STD) during anal sex is higher than that of oral sex $[19,20]$. Therefore, this study focused on the anxiety and depression symptoms differentiated by anal sex roles among MSM. Studies [21] have shown that MSM playing the anal sex role "1" generally have male characteristics and take an active role while MSM playing the anal sex role " 0 " tend to be feminine and take a passive and submissive role in male sexual intercourse. In addition, there are quite a number of MSM playing both the " 1 " and " 0 " roles, generally expressed as the anal sex role " 0.5 ". Therefore, there are considerable sexual role conflicts in MSM [22,23]. Different anal sex roles tend to form different sexual psychology, and the conflicts of sex roles will lead to different mental disorders, especially anxiety and depression, which are the most common symptoms [24]. However, research on anxiety and depression in relation to different anal sex roles in MSM in China are limited.

A growing number of reports [11,25] have revealed that social demographic characteristics, sexual orientation, unprotected sexual behavior, commercial sexual behavior, stigma effect, and so on are related to anxiety and depression symptoms in MSM. Moreover, Parker [26] and Fendrich [27] also suggested that alcohol use and substance use are associated with anxiety and depression. In China, due to the deep-rooted traditional culture, the requirement of society for men is to have a son to carry on his family name. As a result, many MSM have to hide their homosexual orientation through marriage, and unmarried MSM also have to perform homosexual activities underground. In addition, there are studies $[28,29]$ that show that mental disorders of HIV-negative MSM may increase the risk of HIV infection, but research in China and other countries has always focused on the psychological condition of HIV-positive MSM [30-32]. As previous studies [17,33] showed, MSM in different anal sex role groups differed significantly and the risk of HIV infection was higher in the anal sex role " 0 " group and " 0.5 " group. Therefore, it is necessary to distinguish anal sex roles when exploring the influencing factors of anxiety and depression in HIV-negative MSM.

It is worth mentioning that western China (especially Chongqing and Sichuan, known as the "gay capital" in China) is referred to as a high gathering place of MSM and is the focus area of an HIV epidemic while few studies have been conducted on mental health differentiated by anal sex roles in MSM. Therefore, based on the anal sex role of MSM, this study explored the prevalence and influencing factors of anxiety and depression of HIV-negative MSM in four provinces (Chongqing, Xinjiang, Sichuan, and Guangxi) of western China for the first time, which has certain innovative and practical significance for a better understanding of the sexual culture and psychology of MSM in western China. Furthermore, it also provides a basis for formulating prevention and intervention policies and guidelines for the mental health of HIV-negative MSM in China, especially in western China. 


\section{Materials and Methods}

\subsection{Study Procedures}

The data of our study were collected from a prospective cohort study "An open, randomized, multi-center, parallel-controlled clinical intervention trial conducted in western China on MSM taking antiviral drugs to prevent new HIV infections", which was initiated by the Ministry of Science and Technology of China. From April 2013 to March 2015, a total of 2422 participants were recruited using non-probability sampling (including non-governmental organizations (NGOs), peer introduction, core members "snowball", AIDS voluntary counseling and testing (VCT) clinics, network, etc.) in four research sites (Chongqing, Guangxi, Xinjiang, and Sichuan Province). In this study, 1771 MSM met the inclusion and exclusion criteria and completed the baseline survey and anxiety and depression scales.

Inclusion criteria: (a) Signing informed consent; (b) age $\geq 18$ years old and $\leq 65$ years old; (c) HIV negative; (d) average sex activity once every two weeks; and (e) at least one or more male sex couples per month before the trial.

Exclusion criteria: (a) HIV antibody positive; (b) HBsAg (hepatitis B surface antigen) or anti-HBc (hepatitis B core antibody) positive; (c) missing values in male sexual partners in the past 6 months; (d) missing values in the anal sex role; and (e) incomplete anxiety or depression scale.

\subsection{Study Content and Measurements}

\subsubsection{Assessment of Anxiety}

Anxiety was measured using the Self-Rating Anxiety Scale (SAS) compiled by Zung in 1971 [34], having been widely used in clinical trials and psychological evaluation studies. The scale consists of 20 items, including 15 items in the forward direction and 5 items in the reverse direction. The frequency of symptoms was assessed by a four-grade score $(1=$ no or very little time, 2 = a few times, $3=$ quite a lot of times, $4=$ most or all the time). The scores for the 20 items were aggregated into a raw score, and a standard score was obtained by multiplying the raw score by 1.25 and then taking the integer. A standard score equal to or greater than 50 indicated an anxiety symptom and a higher score indicated greater levels of anxiety symptoms. The standardized Cronbach's $\alpha$ in this study was 0.8207 .

\subsubsection{Assessment of Depression}

Depression was assessed with the Center for Epidemiological Studies Depression Scale (CES-D) [35]. The scale consists of 20 items, of which 16 items were positive and 4 items were negative. According to the frequency of symptoms in the last week, the respondents scored, by themselves, from 0 (occasional or no) to 3 (most of the time or persistence). With a total score of 60 points, 16 points or above were identified as having depression symptoms. The standardized Cronbach's $\alpha$ in this study was 0.8192.

\subsubsection{Anal Sex Role Factors}

All participants were requested to report "what is your usual sex position during anal sexual activities with men?" at baseline. In our study, MSM were divided into three anal sex role subgroups: (a) Anal sex role "1" (insertive anal sex) referred to the way that MSM only had insertive anal sex with men; (b) anal sex role " 0 " (receptive anal sex) meant that MSM only played a receptive anal sex role with male sex partners; and (c) anal sex role " 0.5 " (versatile anal sex) indicated men who engaged equally in both insertive and receptive anal sex intercourse with men, also expressed as a versatile anal sex role.

\subsubsection{Social Demographic Factors}

Social demographic factors mainly included age, urban or rural areas, ethnic groups, educational level, employment status, marital status, and personal monthly income. 


\subsubsection{Behavior-Related Factors}

Behavior-related factors mainly included sexual behaviors and substance use behaviors. Sexual behaviors of MSM in this study included the number of regular male sexual partners in the past 6 months, the number of casual male sexual partners in the past 6 months, the number of female sexual partners in the past 6 months, and the frequency of seeking sexual partners on the Internet in the past 6 months (often, sometimes or occasionally, never), whether commercial sexual service has occurred in the past 6 months, and diagnosed with an STD in the past six months. Substance use included the frequency of alcohol use in the last month (daily, occasionally, never), and the use of illicit drugs.

\subsubsection{Risk Perception-Related Factors}

In this study, the definition of risk perception was to perceive the risk of HIV transmission processes, including the source, route, and susceptible population in the MSM population [36,37]. Risk perception values of the source of HIV infection included: Perceived severity of AIDS (very serious, serious, general, not serious, not serious at all), perceived prevalence of HIV among MSM in the participant's city (very high, high, general, low, very low), and perceived threat of HIV to the participant and their family (very large, relatively large and moderate, very small). Risk perception values of the transmission route included: The frequency of condom use when having anal sex with men (always, occasionally, never). Risk perception values of the susceptible population included the following question: Have you ever engaged in HIV voluntary counseling and testing (VCT)? (no, yes).

\subsection{Quality Control and Ethics}

Based on a large number of studies, this research plan was formulated and demonstrated by experts in infectious diseases, epidemiology, and health statistics. Investigators and quality controllers were trained strictly, and the logicality and integrity of the questionnaire content were checked. This study followed the Helsinki Declaration and was approved by the Ethics Committee of Chongqing Medical University. In addition, the experiment was supervised by the ethics committee, and the participants all signed the informed consent.

\subsection{Statistical Analysis}

The database was established by the Epidata 3.1 software (EpiData Associations, Odense, Denmark), and real-time double entry and logical verification of the data were carried out. Statistical analysis was performed by the SAS 9.4 software (SAS Institute, Cary, NC, USA). The score for anxiety and depression was described by mean $\pm \mathrm{SD}$ (standard deviation) and the median. Anxiety and depression scores of different anal sex roles were compared by analysis of variance (ANOVA). Univariate analysis of anxiety and depression was performed by the $\chi 2$ test and Fisher's exact test. The variables were screened using stepwise regression in multivariate logistic regression analysis (sle $=0.05$, sls $=0.05)$, and the odds ratio $(\mathrm{OR})$ value and $95 \%$ confidence intervals $(\mathrm{CI})$ were calculated. $p<$ 0.05 was considered statistically significant. In addition, the average missing rate of variables was below $0.8 \%$, and observations with missing values were excluded from the analyses (Table 1 ). 
Table 1. Sample characteristics of human immunodeficiency virus negative (HIV-negative) men who have sex with men (MSM) and univariate analysis.

\begin{tabular}{|c|c|c|c|c|c|c|c|}
\hline \multirow{2}{*}{ Characteristics } & \multirow{2}{*}{ Total $n(\%)$} & \multicolumn{2}{|c|}{ Anxiety } & \multirow{2}{*}{$p$ Value } & \multicolumn{2}{|c|}{ Depression } & \multirow{2}{*}{$p$ Value } \\
\hline & & NO $n(\%)$ & YES $n(\%)$ & & NO $n(\%)$ & YES $n(\%)$ & \\
\hline$N$ & $1771(100.00)$ & $1310(73.97)$ & $461(26.03)$ & & $1101(62.17)$ & $670(37.83)$ & \\
\hline \multicolumn{8}{|l|}{ Anal sex role factors } \\
\hline \multicolumn{8}{|c|}{ The role of MSM in anal sex with male sexual partners } \\
\hline Anal sex role "1" & $467(26.37)$ & $363(77.73)$ & $104(22.27)$ & 0.0415 & $314(67.24)$ & $153(32.76)$ & 0.0047 \\
\hline Anal sex role “ 0.5 " & $1122(63.35)$ & $822(73.26)$ & $300(26.74)$ & & $689(61.41)$ & $433(38.59)$ & \\
\hline Anal sex role “0” & $182(10.28)$ & $125(68.68)$ & $57(31.32)$ & & $98(53.85)$ & $84(46.15)$ & \\
\hline \multicolumn{8}{|l|}{ Social demographic factors } \\
\hline \multicolumn{8}{|l|}{ Age } \\
\hline $18-24$ & $557(31.45)$ & $400(71.81)$ & $157(28.19)$ & 0.1633 & $321(57.63)$ & $236(42.37)$ & 0.0285 \\
\hline $25-30$ & $532(30.04)$ & $389(73.12)$ & $143(26.88)$ & & $342(64.29)$ & $190(35.71)$ & \\
\hline$\geq 31$ & $682(38.51)$ & $521(76.39)$ & $161(23.61)$ & & $438(64.22)$ & $244(35.78)$ & \\
\hline \multicolumn{8}{|l|}{ Area \# } \\
\hline Urban & $1267(71.74)$ & $971(76.64)$ & $296(23.26)$ & $<0.0001$ & $829(65.43)$ & $438(34.57)$ & $<0.0001$ \\
\hline Rural & $499(28.26)$ & $337(67.54)$ & $162(32.46)$ & & $271(54.31)$ & $228(45.69)$ & \\
\hline \multicolumn{8}{|l|}{ Ethnic groups \# } \\
\hline Han nationality & $1638(92.54)$ & $1201(73.32)$ & $437(26.68)$ & 0.0324 & $1009(61.60)$ & $629(38.40)$ & 0.0944 \\
\hline Other ethnic minorities & $132(7.46)$ & $108(81.82)$ & $24(18.18)$ & & $91(68.94)$ & $41(31.06)$ & \\
\hline \multicolumn{8}{|l|}{ Educational level ${ }^{\#}$} \\
\hline Junior high or below & $215(12.15)$ & $128(59.53)$ & $87(40.47)$ & $<0.0001$ & $108(50.23)$ & 107 (49.77) & $<0.0001$ \\
\hline Senior high & $468(26.46)$ & $330(70.51)$ & $138(29.49)$ & & $275(58.76)$ & $193(41.24)$ & \\
\hline College or graduate or above & $1086(61.39)$ & $850(78.27)$ & $236(21.73)$ & & $716(65.93)$ & $370(34.07)$ & \\
\hline \multicolumn{8}{|l|}{ Employment status \# } \\
\hline On the job & $1358(76.81)$ & $1016(74.82)$ & $342(25.18)$ & 0.0150 & $861(63.40)$ & $497(36.60)$ & 0.1219 \\
\hline Students at school & $243(13.74)$ & $184(75.72)$ & $59(24.28)$ & & $144(59.26)$ & $99(40.74)$ & \\
\hline Retired or unemployed & $167(9.45)$ & $108(64.67)$ & $59(35.33)$ & & $94(56.29)$ & $73(43.71)$ & \\
\hline \multicolumn{8}{|l|}{ Marital status } \\
\hline Unmarried & $1314(74.20)$ & $986(75.04)$ & $328(24.96)$ & 0.0706 & $834(63.47)$ & $480(36.53)$ & 0.1257 \\
\hline Married & $307(17.33)$ & $211(68.73)$ & $96(31.27)$ & & $176(57.33)$ & $131(42.67)$ & \\
\hline Divorce or widowhood & $150(8.47)$ & $113(75.33)$ & $37(24.67)$ & & $91(60.67)$ & $59(39.33)$ & \\
\hline
\end{tabular}


Table 1. Cont

\begin{tabular}{|c|c|c|c|c|c|c|c|}
\hline \multirow{2}{*}{ Characteristics } & \multirow{2}{*}{ Total $n(\%)$} & \multicolumn{2}{|c|}{ Anxiety } & \multirow{2}{*}{$p$ Value } & \multicolumn{2}{|c|}{ Depression } & \multirow{2}{*}{$p$ Value } \\
\hline & & NO $n(\%)$ & YES $n(\%)$ & & NO $n(\%)$ & YES $n(\%)$ & \\
\hline \multicolumn{8}{|l|}{ Personal monthly income $\#$} \\
\hline$\leq 3000$ & $927(52.94)$ & $658(70.98)$ & $269(29.02)$ & 0.0014 & $522(56.31)$ & 405 (43.69) & $<0.0001$ \\
\hline $3001-5000$ & $602(34.38)$ & $454(75.42)$ & $148(24.58)$ & & $398(66.11)$ & $204(33.89)$ & \\
\hline$\geq 5001$ & $222(12.68)$ & $183(82.43)$ & $39(17.57)$ & & $168(75.68)$ & $54(24.32)$ & \\
\hline \multicolumn{8}{|l|}{ Behavior-related factors } \\
\hline \multicolumn{8}{|c|}{ Regular male sexual partners in the last six months \# } \\
\hline 0 & $293(16.80)$ & $217(74.06)$ & $76(25.94)$ & 0.2290 & $179(61.09)$ & $114(38.91)$ & 0.3778 \\
\hline 1 & $1188(68.12)$ & $892(75.08)$ & $296(24.92)$ & & $755(63.55)$ & $433(36.45)$ & \\
\hline$\geq 2$ & $263(15.08)$ & $184(69.96)$ & $79(30.04)$ & & $156(59.32)$ & $107(40.68)$ & \\
\hline \multicolumn{8}{|c|}{ Casual male sexual partners in the last six months \# } \\
\hline 0 & $705(40.68)$ & $541(76.74)$ & $164(23.26)$ & 0.0373 & $466(66.10)$ & $239(33.90)$ & 0.0072 \\
\hline$\geq 1$ & $1028(59.32)$ & $743(72.28)$ & $285(27.72)$ & & $614(59.73)$ & $414(40.27)$ & \\
\hline \multicolumn{8}{|c|}{ Female sexual partners in the last six months \# } \\
\hline 0 & $1462(84.17)$ & $1113(76.13)$ & $349(23.87)$ & $<0.0001$ & $944(64.57)$ & $518(35.43)$ & $<0.0001$ \\
\hline$\geq 1$ & $275(15.83)$ & $172(62.55)$ & $103(37.45)$ & & $137(49.82)$ & $138(50.18)$ & \\
\hline \multicolumn{8}{|c|}{$\begin{array}{c}\text { Frequency of seeking partners on the Internet in the last } 6 \\
\text { months }\end{array}$} \\
\hline Often & $117(6.62)$ & $69(58.97)$ & $48(41.03)$ & 0.0006 & $55(47.01)$ & $62(52.99)$ & 0.0008 \\
\hline Sometimes or occasionally & $986(55.80)$ & $743(75.35)$ & $243(24.65)$ & & $612(62.07)$ & $374(37.93)$ & \\
\hline Never & $664(37.58)$ & 495 (74.55) & $169(25.45)$ & & $434(65.36)$ & $230(34.64)$ & \\
\hline \multicolumn{8}{|c|}{ Whether a commercial sexual service in the past 6 months \# } \\
\hline Yes & $100(5.66)$ & $63(63.00)$ & $37(37.00)$ & 0.0095 & $54(54.00)$ & $46(46.00)$ & 0.0810 \\
\hline No & $1668(94.34)$ & $1246(74.70)$ & $422(25.30)$ & & $1046(62.71)$ & $622(37.29)$ & \\
\hline \multicolumn{8}{|c|}{$\begin{array}{l}\text { Diagnosed with an sexually transmitted diseases (STD) in the } \\
\text { past six months }\end{array}$} \\
\hline $\begin{array}{ll}\text { Yes } \\
\end{array}$ & $142(8.03)$ & $87(61.27)$ & $55(38.73)$ & 0.0003 & $70(49.30)$ & $72(50.70)$ & 0.0009 \\
\hline No & $1626(91.97)$ & $1221(75.09)$ & $405(24.91)$ & & $1030(63.25)$ & $596(36.65)$ & \\
\hline \multicolumn{8}{|c|}{ Frequency of alcohol use in the last month \# } \\
\hline Daily & $72(4.07)$ & $45(62.50)$ & $27(37.50)$ & 0.0464 & $30(41.67)$ & $42(58.33)$ & 0.0009 \\
\hline Occasionally & $1152(65.12)$ & $866(75.17)$ & $286(24.83)$ & & $733(63.63)$ & $419(36.37)$ & \\
\hline Never & $545(30.81)$ & $397(72.84)$ & $148(27.16)$ & & $336(61.65)$ & $209(38.35)$ & \\
\hline
\end{tabular}


Table 1. Cont.

\begin{tabular}{|c|c|c|c|c|c|c|c|}
\hline \multirow{2}{*}{ Characteristics } & \multirow{2}{*}{ Total $n(\%)$} & \multicolumn{2}{|c|}{ Anxiety } & \multirow{2}{*}{$p$ Value } & \multicolumn{2}{|c|}{ Depression } & \multirow{2}{*}{$p$ Value } \\
\hline & & NO $n(\%)$ & YES $n(\%)$ & & NO $n(\%)$ & YES $n(\%)$ & \\
\hline \multicolumn{8}{|c|}{ Whether a use of illicit drugs in the past six months \# } \\
\hline No & $1695(96.91)$ & $1261(74.40)$ & $434(25.60)$ & 0.1136 & $1058(62.42)$ & $637(37.58)$ & 0.8452 \\
\hline Yes & $54(3.09)$ & $35(64.81)$ & $19(35.19)$ & & $33(61.11)$ & $21(38.89)$ & \\
\hline \multicolumn{8}{|l|}{ Risk perception-related factors } \\
\hline \multicolumn{8}{|l|}{ Perceived severity of AIDS \# } \\
\hline Very serious & $1107(62.54)$ & $832(75.16)$ & $275(24.84)$ & 0.3072 * & $679(61.34)$ & $428(38.66)$ & 0.5051 * \\
\hline Serious & $522(29.49)$ & $379(72.61)$ & $143(27.39)$ & & $334(63.98)$ & $188(36.02)$ & \\
\hline General & $123(6.95)$ & $87(70.73)$ & $36(29.27)$ & & $77(62.60)$ & $46(37.40)$ & \\
\hline Not serious & $10(0.56)$ & $7(70.00)$ & $3(30.00)$ & & $7(70.00)$ & $3(30.00)$ & \\
\hline Not serious at all & $8(0.45)$ & $4(50.00)$ & $4(50.00)$ & & $3(37.50)$ & $5(62.50)$ & \\
\hline \multicolumn{8}{|c|}{ Perceived prevalence of HIV among MSM in your city \# } \\
\hline Very high & $375(21.23)$ & $258(68.80)$ & $117(31.20)$ & 0.0691 & $219(58.40)$ & $156(41.60)$ & 0.2287 \\
\hline High & $774(43.83)$ & $595(76.87)$ & $179(23.13)$ & & $502(64.86)$ & $272(35.14)$ & \\
\hline General & $420(23.78)$ & $308(73.33)$ & $112(26.67)$ & & $253(60.24)$ & $167(39.76)$ & \\
\hline Low & $126(7.13)$ & $93(73.81)$ & $33(26.19)$ & & $81(64.29)$ & $45(35.71)$ & \\
\hline Very low & $71(4.02)$ & $52(73.24)$ & $19(26.76)$ & & $43(60.56)$ & $28(39.44)$ & \\
\hline \multicolumn{8}{|c|}{ Perceived threat of HIV to yourself and your family \# } \\
\hline Very large & $973(55.03)$ & $707(72.66)$ & $266(27.34)$ & 0.0994 & $587(60.33)$ & $386(39.67)$ & 0.0147 \\
\hline Relatively large and moderate & $639(36.14)$ & $474(74.18)$ & $165(25.82)$ & & $400(62.60)$ & $239(37.40)$ & \\
\hline Very small & $156(8.82)$ & $126(80.77)$ & $30(19.23)$ & & $113(72.44)$ & $43(27.56)$ & \\
\hline \multicolumn{8}{|c|}{ Frequency of condom use when having anal sex with men ${ }^{\#}$} \\
\hline Always & $956(56.84)$ & $725(75.84)$ & $231(24.16)$ & 0.0051 & $616(64.44)$ & $340(35.56)$ & 0.0050 \\
\hline Occasionally & $562(33.41)$ & $423(75.27)$ & $139(24.73)$ & & $356(63.35)$ & $206(36.65)$ & \\
\hline Never & $164(9.75)$ & $105(64.02)$ & $59(35.98)$ & & $84(51.22)$ & $80(48.78)$ & \\
\hline \multicolumn{8}{|l|}{ HIV voluntary counseling \# } \\
\hline Yes & $1074(60.88)$ & $820(76.35)$ & $254(23.65)$ & 0.0046 & 719 (66.95) & $355(33.05)$ & $<0.0001$ \\
\hline No & $690(39.12)$ & $485(70.29)$ & $205(29.71)$ & & $377(54.64)$ & $313(45.36)$ & \\
\hline \multicolumn{8}{|l|}{ HIV voluntary testing \# } \\
\hline Yes & $1362(77.17)$ & $1021(74.96)$ & $341(25.04)$ & 0.1586 & $870(63.88)$ & $492(36.12)$ & 0.0133 \\
\hline No & $403(22.83)$ & $288(71.46)$ & $115(28.54)$ & & $230(57.07)$ & $173(42.93)$ & \\
\hline
\end{tabular}

* Fisher's exact test and $\chi^{2}$ test was used for the rest. ${ }^{*}$ indicates loss of data. Bold values indicate statistical significance at $p<0.05$. 


\section{Results}

\subsection{Participants' Characteristics}

A total of 2422 subjects were recruited from Chongqing, Sichuan, Guangxi, and Xinjiang in western China, and 1771 HIV-negative MSM were eligible (Figure 1). Among them, 467 (26.37\%) MSM were classified as anal sex role "1", $182(10.28 \%)$ as anal sex role " 0 ", and $1122(63.35 \%)$ as anal sex role " 0.5 ". The average age of the participants was 29.89 years (median $=28$ ), that of anal sex role " 1 " was 32.02 years (median $=31$ ), anal sex role " 0 " was 26.79 years (median $=25$ ), and anal sex role " 0.5 " was 29.51 years (median $=27$ ). Urban and rural MSM accounted for $71.71 \%$ and $28.26 \%$, respectively. The majority of MSM were well educated, of which $61.39 \%$ had a college or undergraduate degree or above, $26.46 \%$ had a high school or professional high school degree, and only $12.15 \%$ had a junior high school or below. A total of $76.81 \%$ of MSM had a job, $9.45 \%$ were retired or unemployed, and $13.74 \%$ were students. Unmarried MSM accounted for 74.20\%. Almost half (52.94\%) of the participants had an average monthly income of 3000 yuan or less, 34.38\% between 3001 and 5000 yuan, and only 12.68\% over 5001 yuan (Table 1).

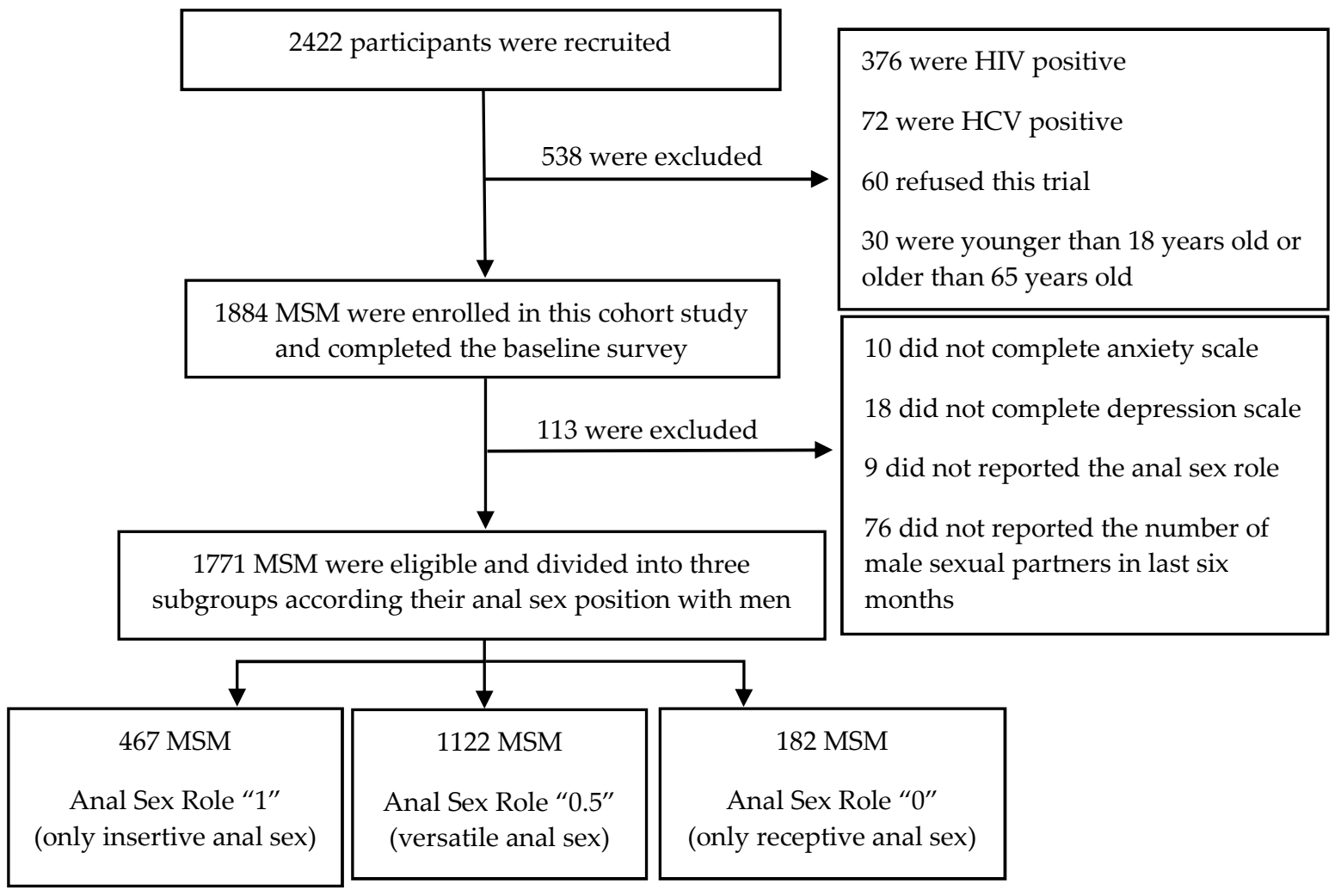

Figure 1. Flow chart of participants' enrollment.

\subsection{The Prevalence of Anxiety and Depression in Different Anal Sex Roles of MSM}

There were 461 (26.03\%) HIV-negative MSM who experienced anxiety and $670(37.83 \%)$ HIV-negative MSM who experienced depression in western China (Table 1). The anxiety and depression scores of different anal sex role groups are shown in Table 2. Among all the participants, the mental problems of HIV-negative MSM in anal sex role " 0 " were the most serious, and the prevalence of anxiety and depression were $31.32 \%$ and $46.15 \%$, respectively. The prevalence of anxiety and depression in the anal sex role " 0.5 " group followed, and that of the anal sex role " 1 " group was the lowest. Moreover, the $\chi^{2}$ test suggested that the prevalence of anxiety and depression was significantly different among the anal sex role groups $(p<0.05)$ (Table 2$)$. 
Table 2. Scores and prevalence of anxiety and depression in different anal sex roles.

\begin{tabular}{|c|c|c|c|c|c|}
\hline \multirow[t]{2}{*}{ Mental Health Problems } & $\begin{array}{l}\text { Anal Sex Role } \\
\text { "1" }(n=467)\end{array}$ & $\begin{array}{l}\text { Anal Sex Role } \\
\text { “0.5” }(n=1122)\end{array}$ & $\begin{array}{l}\text { Anal Sex Role } \\
\text { “0” }(n=182)\end{array}$ & \multirow[t]{2}{*}{$F / \chi^{2}$} & \multirow[t]{2}{*}{$p$} \\
\hline & Score/N (\%) & Score/N (\%) & Score/N (\%) & & \\
\hline \multicolumn{6}{|l|}{ Anxiety } \\
\hline Mean \pm SD & $42.09 \pm 10.20$ & $42.94 \pm 10.75$ & $44.25 \pm 11.55$ & 2.80 & $0.0611^{\mathrm{a}}$ \\
\hline Median & 42.00 & 42.00 & 42.00 & & \\
\hline Yes $(\geq 50)$ & $104(22.27)$ & $300(26.74)$ & $57(31.32)$ & 6.3652 & $0.0415^{b}$ \\
\hline No $(<50)$ & $363(77.73)$ & $822(73.26)$ & $125(68.68)$ & & \\
\hline \multicolumn{6}{|l|}{ Depression } \\
\hline Mean \pm SD & $12.78 \pm 8.93$ & $14.02 \pm 9.62$ & $16.31 \pm 11.71$ & 8.83 & $0.0002^{a}$ \\
\hline Median & 12.00 & 12.00 & 13.50 & & \\
\hline Yes $(\geq 16)$ & $153(32.76)$ & $433(38.59)$ & $84(46.15)$ & 10.7378 & $0.0047^{b}$ \\
\hline No $(<16)$ & $314(67.24)$ & $689(61.41)$ & $98(53.85)$ & & \\
\hline
\end{tabular}

\subsection{The Influencing Factors of Anxiety in HIV-Negative MSM}

In the univariate analysis, significant differences in the prevalence of anxiety were observed between groups with different characteristics, including anal sex roles, urban or rural, ethnic groups, educational level, employment status, average monthly income, casual male partners, female partners, frequency of seeking partners on the Internet, commercial sexual service, diagnosed with an STD, alcohol use, perceived threat of HIV, condom use, and HIV counseling $(p<0.05)$ (Table 1).

In the multivariate model (Table 3), anxiety in HIV-negative MSM was associated with anal sex role " 0 " or " 0.5 ", low educational level, female sexual partners, frequently seeking sexual partners on the Internet, STD diagnosis, and no HIV counseling $(p<0.05)$. Anal sex role " 0 " had the highest risk of anxiety, which was 1.783 times higher than anal sex role "1" (OR 1.783, 95\% CI 1.188-2.677), and anal sex role " 0.5 " had a 1.336 times higher risk of anxiety than " 1 " (OR 1.336, 95\% CI 1.021-1.750). Compared with HIV-negative MSM who had a bachelor or graduate degree or above, MSM under junior high school or in senior high school were more likely to experience anxiety (OR 2.276, 95\% CI 1.643-3.154; OR 1.366, 95\% CI 1.054-1.770, respectively). Furthermore, having female partners in the past six months (OR 1.717, 95\% CI 1.285-2.293) or often searching for partners on the Internet (OR $1.793,95 \%$ CI 1.163-2.763) also increased the risk of anxiety. Moreover, MSM who were diagnosed with an STD by a doctor were more likely to suffer from anxiety (OR 1.813, 95\% CI 1.242-2.649). However, HIV counseling showed to be a protective factor against anxiety for HIV-negative MSM (OR 0.769, 95\% CI 0.613-0.965).

Table 3. Multivariate logistic stepwise regression of anxiety in HIV-negative MSM.

\begin{tabular}{|c|c|c|c|c|c|c|c|}
\hline \multirow{2}{*}{ Independent Variables } & \multirow{2}{*}{ B } & \multirow{2}{*}{ S.E. } & \multirow{2}{*}{ Wald } & \multirow{2}{*}{$p$ Value } & \multirow{2}{*}{ OR } & \multicolumn{2}{|c|}{$95 \% \mathrm{CI}$ for OR } \\
\hline & & & & & & Lower & Upper \\
\hline $\begin{array}{l}\text { Anal sex role } \\
\text { "1" }\end{array}$ & & & & & & & (reference) \\
\hline “0.5” & 0.2900 & 0.1374 & 4.4534 & 0.0348 & 1.336 & 1.021 & 1.750 \\
\hline “0” & 0.5783 & 0.2073 & 7.7802 & 0.0053 & 1.783 & 1.188 & 2.677 \\
\hline Educational level & & & & & & & \\
\hline College or graduate or above & & & & & & & (reference) \\
\hline Senior high & 0.3117 & 0.1322 & 5.5604 & 0.0184 & 1.366 & 1.054 & 1.770 \\
\hline Junior high or below & 0.8225 & 0.1664 & 24.4415 & $<0.0001$ & 2.276 & 1.643 & 3.154 \\
\hline
\end{tabular}


Table 3. Cont.

\begin{tabular}{|c|c|c|c|c|c|c|c|}
\hline \multirow{2}{*}{ Independent Variables } & \multirow{2}{*}{ B } & \multirow{2}{*}{ S.E. } & \multirow{2}{*}{ Wald } & \multirow{2}{*}{$p$ Value } & \multirow{2}{*}{ OR } & \multicolumn{2}{|c|}{$95 \%$ CI for OR } \\
\hline & & & & & & Lower & Upper \\
\hline \multicolumn{8}{|l|}{$\begin{array}{l}\text { Female sexual partners in the } \\
\text { last six months }\end{array}$} \\
\hline 0 & & & & & & & (reference) \\
\hline$\geq 1$ & 0.5404 & 0.1478 & 13.3661 & 0.0003 & 1.717 & 1.285 & 2.293 \\
\hline \multicolumn{8}{|l|}{$\begin{array}{l}\text { Frequency of seeking } \\
\text { partners on the Internet in } \\
\text { the last } 6 \text { months }\end{array}$} \\
\hline Sometimes or occasionally & -0.0636 & 0.1221 & 0.2715 & 0.6023 & 0.938 & 0.739 & 1.192 \\
\hline Often & 0.5836 & 0.2207 & 6.9947 & 0.0082 & 1.793 & 1.163 & 2.763 \\
\hline \multicolumn{8}{|l|}{$\begin{array}{l}\text { Diagnosed with STD in the } \\
\text { past six months }\end{array}$} \\
\hline No & & & & & & & (reference) \\
\hline Yes & 0.5953 & 0.1933 & 9.4873 & 0.0021 & 1.813 & 1.242 & 2.649 \\
\hline \multicolumn{8}{|l|}{ HIV voluntary counseling } \\
\hline No & & & & & & & (reference) \\
\hline Yes & -0.2628 & 0.1159 & 5.1445 & 0.0233 & 0.769 & 0.613 & 0.965 \\
\hline
\end{tabular}

Dependent variable $=$ anxiety. Bold values indicate statistical significance at $p<0.05$. Statistically significant variables $(p<0.05)$ in univariate analysis were included in the model, and variables with multiple collinearity with anal sex role were excluded: Age, marital status, and personal monthly income.

\subsection{The Influencing Factors of Depression in HIV-Negative MSM}

Univariate analysis revealed that the depression of HIV-negative MSM showed a significant difference in anal sex roles, age groups, urban or rural areas, educational level, average monthly income, casual male partners, female partners, frequency of seeking partners on the Internet, diagnosed with an STD, alcohol use, condom use, perceived threat of HIV, and HIV counseling and testing $(p<0.05)$ (Table 1).

Multivariate logistic regression analysis (Table 4) showed that the prevalence of depression was related to anal sex roles " 0 " and " 0.5 ", rural area, casual male partners, female partners, diagnosed with an STD, frequently seeking partners on the Internet, no HIV counseling, no condom use, and daily alcohol use $(p<0.05)$. Similar to anxiety, anal sex role " 0 " and anal sex role " 0.5 " were exposure factors for depression, and the probability of risk for depression was 1.784 times and 1.309 times that of anal sex role "1" (OR 1.784, 95\% CI 1.212-2.625; OR 1.309, 95\% CI 1.019-1.682, respectively). Furthermore, the risk of depression in urban HIV-negative MSM was lower than that in rural HIV-negative MSM (OR $0.662,95 \%$ CI 0.525-0.834). In terms of behavior-related factors, MSM with casual male partners (OR 1.319, 95\% CI 1.042-1.670) or female partners (OR 1.721, 95\% CI: 1.291-2.295) in the past six months increased the appearance of a depression symptom. MSM who were diagnosed with an STD were more likely to be depressed as well (OR 1.673, 95\% CI 1.143-2.447). MSM who often sought partners through the Internet in the past six months were 1.947 times more likely to develop depression than those of MSM never finding any online partners (OR 1.947, 95\% CI 1.244-3.048). In addition, the model also implied that MSM who drank alcohol every day in the most recent month were more likely to be depressed than MSM who never drank (OR 2.362, 95\% CI 1.385-4.029). In regards to risk perception-related factors, compared with MSM without condom use, always or occasional condom use were protective factors for depression in HIV-negative MSM (OR 0.636, 95\% CI 0.441-0.918; OR $0.598,95 \%$ CI $0.407-0.879$, respectively). Furthermore, the risk of depression in HIV-negative MSM would be significantly decreased when someone had ever received HIV voluntary counseling (OR $0.588,95 \%$ CI $0.473-0.730$ ). 
Table 4. Multivariate logistic stepwise regression of depression in HIV-negative MSM.

\begin{tabular}{|c|c|c|c|c|c|c|c|}
\hline \multirow{2}{*}{ Independent Variables } & \multirow{2}{*}{ B } & \multirow{2}{*}{ S.E. } & \multirow{2}{*}{ Wald } & \multirow{2}{*}{$p$ Value } & \multirow{2}{*}{ OR } & \multicolumn{2}{|c|}{$95 \% \mathrm{CI}$ for OR } \\
\hline & & & & & & Lower & Upper \\
\hline \multicolumn{8}{|l|}{ Anal sex role } \\
\hline “0.5” & 0.2693 & 0.1278 & 4.4395 & 0.0351 & 1.309 & 1.019 & 1.682 \\
\hline “0” & 0.5787 & 0.1971 & 8.6243 & 0.0033 & 1.784 & 1.212 & 2.625 \\
\hline \multicolumn{8}{|l|}{ Area } \\
\hline Rural & & & & & & & (reference) \\
\hline Urban & -0.4127 & 0.1182 & 12.1885 & 0.0005 & 0.662 & 0.525 & 0.834 \\
\hline \multicolumn{8}{|l|}{$\begin{array}{l}\text { Casual sexual partners in the } \\
\text { last six months }\end{array}$} \\
\hline 0 & & & & & & & (reference) \\
\hline$\geq 1$ & 0.2772 & 0.1202 & 5.3133 & 0.0212 & 1.319 & 1.042 & 1.670 \\
\hline \multicolumn{8}{|l|}{$\begin{array}{l}\text { Female sexual partners in the } \\
\text { last six months }\end{array}$} \\
\hline 0 & & & & & & & (reference) \\
\hline$\geq 1$ & 0.5432 & 0.1467 & 13.7131 & 0.0002 & 1.721 & 1.291 & 2.295 \\
\hline \multicolumn{8}{|l|}{$\begin{array}{l}\text { Diagnosed with STD in the } \\
\text { past six months }\end{array}$} \\
\hline No & & & & & & & (reference) \\
\hline Yes & 0.5144 & 0.1941 & 7.0253 & 0.0080 & 1.673 & 1.143 & 2.447 \\
\hline \multicolumn{8}{|l|}{$\begin{array}{l}\text { Frequency of seeking } \\
\text { partners on the Internet in } \\
\text { the last } 6 \text { months }\end{array}$} \\
\hline Never & & & & & & & (reference) \\
\hline Sometimes or occasionally & 0.0124 & 0.1233 & 0.0101 & 0.9198 & 1.012 & 0.795 & 1.289 \\
\hline Often & 0.6664 & 0.2287 & 8.4909 & 0.0036 & 1.947 & 1.244 & 3.048 \\
\hline \multicolumn{8}{|l|}{$\begin{array}{l}\text { Frequency of alcohol use in } \\
\text { the last month }\end{array}$} \\
\hline Never & & & & & & & (reference) \\
\hline Occasionally & -0.1704 & 0.1177 & 2.0954 & 0.1477 & 0.843 & 0.670 & 1.062 \\
\hline Daily & 0.8594 & 0.2725 & 9.9499 & 0.0016 & 2.362 & 1.385 & 4.029 \\
\hline \multicolumn{8}{|l|}{$\begin{array}{l}\text { Frequency of condom use } \\
\text { when having anal sex with } \\
\text { men }\end{array}$} \\
\hline Never & & & & & & & (reference) \\
\hline Occasionally & -0.5143 & 0.1966 & 6.8456 & 0.0089 & 0.598 & 0.407 & 0.879 \\
\hline Always & -0.4524 & 0.1871 & 5.8425 & 0.0156 & 0.636 & 0.441 & 0.918 \\
\hline \multicolumn{8}{|l|}{ HIV voluntary counseling } \\
\hline No & & & & & & & (reference) \\
\hline Yes & -0.5317 & 0.1104 & 23.1937 & $<0.0001$ & 0.588 & 0.473 & 0.730 \\
\hline
\end{tabular}

\section{Discussion}

\subsection{The Prevalence of Anxiety and Depression in HIV-Negative MSM}

This study found that mental health problems were common in HIV-negative MSM of western China, and the prevalence of anxiety and depression was not optimistic, especially for MSM having sexual intercourse with men in anal sex role " 0 " and anal sex role " 0.5 ".

The prevalence of anxiety in HIV-negative MSM in four western provinces of China was 26.03\%, slightly higher than that in Zhejiang Province (24.0\%) as the developed area of eastern China [15], and 
significantly higher than that in Guangzhou City (14.5\%) as the developed city of southern China [30]. It suggests that when compared with the developed coastal cities in eastern or southern China, MSM of western China show a rather high prevalence level, perhaps due to a relatively backward economy, a less open culture, and less social support. Furthermore, the prevalence of anxiety in the HIV-negative MSM of our study was lower than that in Chongqing (37.5\%) but similar to that in Sichuan $(24.0 \%)$ in HIV-positive MSM of a previous study. This also indicates that the prevalence of anxiety in HIV-negative MSM is relatively lower than HIV-positive MSM [30] but still at a higher prevalence level. Although few reports have been done on anxiety symptoms in HIV-negative MSM in international studies, studies conducted in Vancouver, Canada [12], and Estonia [26] share similar opinions with ours and have reported a high prevalence as well.

In terms of depression, our study also reported a high prevalence of depression of $37.83 \%$. Compared with other areas in China, our result in the west is in the upper middle level: $22.4 \%$ in Shenyang, northeastern China [38]; 34.7\% in Guangzhou, southern China [30]; and 45.4\% in Zhejiang, eastern China [15]. Moreover, the prevalence varies from area to area, due to the differences in culture, economics, and the development of local mental health services. On account of the relatively lagging development of the four provinces in the west that we engaged, and the grim situation of HIV in MSM in these areas, our study is a better representation of the occurrence of HIV-negative MSM in western China, and converges with internationally reported levels: 36.7\% in 1367 MSM in Moscow, Russia [39]; one third in eastern Europe [26]; and 44\% in South Africa [40], respectively.

More importantly, our research demonstrated that the mental health condition of HIV-negative MSM varies among the different anal sex roles; however, few studies have compared the anxiety and depression status of MSM with different anal sex roles internationally. Anal sex role " 0 " of HIV-negative MSM in western China reported the highest risk for anxiety and depression, reaching $31.32 \%$ and $46.15 \%$, respectively, which was obviously higher than the average level of MSM [12,14], and even higher than that of anal sex role "0" in Yi's report [16]. It infers that anxiety and depression symptoms in anal sex role " 0 " MSM are more serious than other anal sex roles, let alone the general population. Thus, we should pay more attention to mental health and its prevention for anal sex role " 0 " MSM.

\subsection{Influencing Factors}

In this study, we primarily focused on four factors: Anal sex role factors, social demographic factors, behavior-related factors, and risk perception-related factors. Although numerous studies have demonstrated the contact between demographic factors and mental disorders, we still pointed out that educational level has severely limited the development of mental health, even worse for the mental health of rural MSM. The lack of education restricts access to HIV knowledge and health care services, gradually increasing their psychological burden. Consistent with the findings in the United States [41], Canada [12], and Kunming [14], but contrary to those in Zhejiang rural MSM [15], this study gains a better understanding of MSM in western China, with huge rural areas and a lower level of economy, culture, and health.

Among all the variables in this study, we found that the anal sex role was an important factor affecting both anxiety and depression in HIV-negative MSM. However, most of the existing literature has overlooked differences in mental disorders between different anal sex roles, usually comparing physical problems, such as HIV infection [21]. Multivariate analysis showed that the risk of anxiety and depression in MSM with anal sex role "0" was the highest, which was 1.791 and 1.784 times higher than that of anal sex role "1", respectively. The possible explanation may be that MSM with anal sex role " 0 " are usually in a passive and submissive role in male sexual activities, their psychological and physiological needs cannot be fulfilled, and long-term frustrations are prone to anxiety and depression symptoms. Additionally, anal sex role "0" MSM generally are reported to have a younger age [17], first anal sexual intercourse at an early age [42], and frequent unprotected anal sex, which in turn can explain their "submissive" status and weak self-protection ability. Thus, mental health problems are highlighted in HIV-negative MSM with anal sex role "0". However, MSM with anal sex role "1" are 
always in the "dominant" position, have more abundant experience, a stronger ability of self-protection, and barely develop mental problems. In line with other research [16], the conflicts of the sex psychology of MSM with anal sex role " 0.5 " were prominent, caused by their variability of sexual partners and behaviors. Meanwhile, previous studies [17] in China also pointed out that MSM with anal sex role " 0 " or " 0.5 " had a higher risk of HIV, perianal Human papillomavirus (HPV), and syphilis infection, which also increased stress and regret after anal intercourse, leading to negative emotions of anxiety or depression. Analogous results have been obtained from MSM research in San Francisco, USA [23] and Lima, Peru [33]. Therefore, more attention should be paid to HIV-negative MSM with an anal sex role of " 0 " and " 0.5 " in comprehensive HIV prevention and control strategy in the future.

As the most directly related features of MSM, we found that high-risk sexual behaviors were an important risk factor for anxiety and depression [43]. China is an ancient country with profound ethical traditions. Many Chinese MSM will marry women to cover up their true sexual orientation for the sake of "having a son to carry his family name" or hide their homosexual activities by searching for partners on the Internet or seeking casual sexual partners. Moreover, our study also suggested that we should provide timely treatment and counseling for HIV-negative MSM who have been diagnosed with an STD, so as to reduce the psychological burden and alleviate anxiety and depression. In addition, similar to the report of Chou [12], our results also indicated that HIV-negative MSM who consumed alcohol every day were more likely to suffer from depression, but the causal relationship needs stronger longitudinal research evidence.

It is worth mentioning that our study also found that the higher the risk perception of HIV transmission sources, transmission routes, and susceptible populations, the more likely it is for HIV-negative MSM to develop mental health problems. The risk perception of transmission routes, including condom use in anal sex, was shown to be a protective factor for depression in HIV-negative MSM. Global experience [4] in AIDS prevention and control has proven the effectiveness of condoms in preventing HIV infection. However, in our study, only half of the participants used a condom at any time, up to $10 \%$ of which never used a condom. Moreover, studies of 3217 Kenyans [44] and 91,477 Europeans [45] also reported a high percentage of anal intercourse without a condom in MSM. After sex, HIV-negative MSM who did not use condoms would have doubts about their partner's health and worry about potential HIV infection, and in the long run, depressive symptoms are likely to occur. Studies in eastern Europe [26] and Shenzhen, China [46] also shared the same view with ours that the prevalence of anxiety and depression in HIV-negative MSM who did not use condoms was even higher. In addition, as the highlight of our study, the passive status in sex activities of the anal sex role " 0 " group restricted the use of condoms, so the proportion of condom use in the " 0 " group was lower and the risk of anxiety and depression was higher [47,48]. In terms of the risk perception of susceptible groups, MSM with HIV counseling were not likely to experience anxiety and depression, which is consistent with the view of David [26]. It revealed that HIV counseling for MSM could give professional advice to understand their own situation and susceptibility in sexual activities, and help to make appropriate psychological construction and adjustment. In addition, although variables of infection sources of risk perception did not enter the regression model, more propaganda and health education will also be needed to alleviate the "fear of AIDS" in the future.

\subsection{Limitation}

It is important to note several limitations in our study. Notably, due to the particularity of the MSM, the study subjects may have a selection bias by using non-probability sampling methods. Meanwhile, this study was not exactly a randomized trial, and it could be called a real-world study, so there is potential bias of the population. Moreover, all sexual practices were self-reported by the respondents, and authenticity could not be determined. There would be some judgment and potential bias of the practices. Moreover, only cross-sectional data of the baseline in the cohort study were used, so the result obtained could not identify a causal relationship. Thus, longitudinal studies are needed in the future. 


\section{Conclusions}

This study found that the prevalence of anxiety and depression in HIV-negative MSM in western China was relatively high, and that of anal sex role " 0 " MSM was the highest. However, few studies have focused on the mental health of different anal sex roles in HIV-negative MSM, so such differences should be given more attention in the future in China. At the same time, high-risk behaviors and high-risk perceptions also increased the risk of anxiety and depression in HIV-negative MSM. Therefore, the relevant departments of health should provide differentiated health services and carry out early identification and key intervention for high-risk groups with anxiety and depression symptoms. Anxiety and depression symptoms may have an impact on the mental health of these populations in the long run. Furthermore, in this study, MSM sexual practices of anal sex role "0" (receptive anal sex) were associated with mental health problems, like anxiety and depression symptoms, which require more psychological counseling, as well as the understanding and support of the social mainstream. Moreover, this study also suggested that the government should improve the accessibility of HIV counseling and testing and advocate condom use in western China, so that HIV-negative MSM with low educational levels and MSM in rural areas can also get timely and better health and medical care for anxious and depressive emotions. In addition, previous studies [49-51] also found that the prevalence of anxiety and depression in HIV-negative MSM affects adherence to HIV prevention and treatment, so early identification of anxiety and depression symptoms and exploration of influencing factors in HIV-negative MSM conducted in our study shows particular importance for the mental health of MSM in western China.

Author Contributions: Data curation, J.L.; Formal analysis, J.L.; Investigation, X.Z., B.P., Y.Z., H.L., J.D. and J.Z.; Methodology, J.L. and X.Z.; Project administration, X.Z., Y.Z. and A.H.; Software, J.L.; Supervision, X.Z. and A.H.; Writing-Original draft, J.L.; Writing-Review \& editing, X.Z. and Z.L. All authors have read and agreed to the published version of the manuscript.

Funding: This work was supported by the National Key Project for Infectious Diseases of the Ministry of Science and Technology of China (grant number: 2012ZX10001007-007); and Chongqing Science and Technology Commission (No. cstc2013jcyjA10009).

Acknowledgments: We thank all participants and investigators in Chongqing, Sichuan, Xinjiang and Guangxi provinces in China for their help.

Conflicts of Interest: The authors declare no conflict of interest.

\section{References}

1. Chow, E.P.F.; Lau, J.T.F.; Zhuang, X.; Zhang, X.; Wang, Y.; Zhang, L. HIV Prevalence Trends, Risky Behaviours, and Governmental and Community Responses to the Epidemic among Men Who Have Sex with Men in China. BioMed Res. Int. 2014, 2014, 607261. [CrossRef] [PubMed]

2. Lin, G.E.; Li, D.; Li, P.; Guo, W.; Cui, Y. Population Specific Sentinel Surveillance for HIV Infection, Syphilis and HCV Infection in China, During 2010-2015. Dis. Surveill. 2017, 32, 111-117. (In Chinese)

3. Wu, Z.; Xu, J.; Liu, E.; Mao, Y.; Xiao, Y.; Sun, X.; Liu, Y.; Jiang, Y.; McGoogan, J.M.; Dou, Z.; et al. HIV and syphilis prevalence among men who have sex with men: A cross-sectional survey of 61 cities in China. Clin. Infect. Dis. 2013, 57, 298-309. [CrossRef] [PubMed]

4. UNAIDS. The Gap Report 2014: Gay Men and Other Men Who Have Sex with Men. Available online: https://www.unaids.org/sites/default/files/media_asset/UNAIDS_Gap_report_en.pdf (accessed on 16 June 2019).

5. Safren, S.A.; Thomas, B.E.; Mimiaga, M.J.; Chandrasekaran, V.; Menon, S.; Swaminathan, S.; Mayer, K.H. Depressive symptoms and human immunodeficiency virus risk behavior among men who have sex with men in Chennai, India. Psychol. Health Med. 2009, 14, 705-715. [CrossRef] [PubMed]

6. Bluthenthal, R.N.; Palar, K.; Mendel, P.; Kanouse, D.E.; Corbin, D.E.; Derose, K.P. Attitudes and beliefs related to HIV/AIDS in urban religious congregations: Barriers and opportunities for HIV-related interventions. Soc. Sci. Med. 2012, 74, 1520-1527. [CrossRef] [PubMed] 
7. Jiang, T.; Zhou, X.; Wang, H.; Luo, M.; Pan, X.; Ma, Q.; Chen, L. Psychosocial Factors Associated with Quality of Life in Young Men Who Have Sex with Men Living with HIV/AIDS in Zhejiang, China. Int. J. Environ. Res. Public Health 2019, 16, 2667. [CrossRef]

8. Salomon, E.A.; Mimiaga, M.J.; Husnik, M.J.; Welles, S.L.; Manseau, M.W.; Montenegro, A.B.; Safren, S.A.; Koblin, B.A.; Chesney, M.A.; Mayer, K.H. Depressive Symptoms, Utilization of Mental Health Care, Substance Use and Sexual Risk among Young Men Who Have Sex with Men in Explore: Implications for Age-Specific Interventions. AIDS Behav. 2009, 13, 811-821. [CrossRef] [PubMed]

9. Kessler, R.C.; McGonagle, K.A.; Zhao, S.; Nelson, C.B.; Hughes, M.; Eshleman, S.; Wittchen, H.-U.; Kendler, K.S. Lifetime and 12-Month Prevalence of DSM-III-R Psychiatric Disorders in the United States. Arch. Gen. Psychiatry 1994, 51, 8-19. [CrossRef] [PubMed]

10. Mills, T.C.; Paul, J.; Stall, R.; Pollack, L.; Canchola, J.; Chang, Y.J.; Moskowitz, J.T.; Catania, J.A. Distress and Depression in Men Who Have Sex with Men: The Urban Men's Health Study. Am. J. Psychiatry 2004, 161, 278-285. [CrossRef] [PubMed]

11. Phillips, M.R.; Zhang, J.; Shi, Q.; Song, Z.; Ding, Z.; Pang, S.; Li, X.; Zhang, Y.; Wang, Z. Prevalence, treatment, and associated disability of mental disorders in four provinces in China during 2001-2005: An epidemiological survey. Lancet 2009, 373, 2041-2053. [CrossRef]

12. Chou, F.Y.; Armstrong, H.L.; Wang, L.; Bacani, N.; Lachowsky, N.J.; Patterson, T.L.; Walsh, Z.; Olarewaju, G.; Card, K.G.; Roth, E.A.; et al. A longitudinal analysis of cannabis use and mental health symptoms among gay, bisexual, and other men who have sex with men in Vancouver, Canada. J. Affect. Disord. 2019, 247, 125-133. [CrossRef] [PubMed]

13. Díaz, R.M.; Ayala, G.; Bein, E.; Henne, J.; Marin, B.V. The impact of homophobia, poverty, and racism on the mental health of gay and bisexual Latino men: Findings from 3 US cities. Am. J. Public Health 2001, 91, 927-932. [PubMed]

14. Gao, X.; Zhang, R.; Li, Y.; Ma, Y.; Chen, Y.; Ma, J.; Li, Z.; Jia, M. Depression and Related Influential Factors among Men Who Have Sex with Men in Kunming. Chin. J. AIDS STD 2018, 24, 170-173. (In Chinese)

15. Jiang, T.; Pan, X.; Wang, H.; Zhou, X.; Ma, Q. Depressive, Anxiety Symptoms and Related Influential Factors among Man Who Have Sex with Man in Zhejiang. Chin. J. AIDS STD 2016, 22, 357-360. (In Chinese)

16. Wang, Y.; Li, L.; Zhou, W.; Fan, J.; Zhao, X.; Tang, Y.; He, J.; Liu, J.; Zhou, L.; Zhang, X. Anxiety and Depressive Symptoms and Related Factors in 15-24 Year-Old Young Men Who Have Sex with Men Behavior. Chin. Ment. Health J. 2018, 32, 1017-1024. (In Chinese)

17. Zeng, X.; Zhong, X.; Peng, B.; Zhang, Y.; Kong, C.; Liu, X.; Huang, A. Prevalence and associated risk characteristics of HIV infection based on anal sexual role among men who have sex with men: A multi-city cross-sectional study in Western China. Int. J. Infect. Dis. 2016, 49, 111-118. [CrossRef]

18. Rich, A.J.; Lachowsky, N.J.; Cui, Z.; Sereda, P.; Lal, A.; Moore, D.M.; Hogg, R.S.; Roth, E.A. Event-Level Analysis of Anal Sex Roles and Sex Drug Use among Gay and Bisexual Men in Vancouver, British Columbia, Canada. Arch. Sex. Behav. 2016, 45, 1443-1451. [CrossRef]

19. Carballo-Diéguez, A.; Bauermeister, J.A.; Ventuneac, A.; Dolezal, C.; Balan, I.; Remien, R.H. The Use of Rectal Douches among HIV-Uninfected and Infected Men Who Have Unprotected Receptive Anal Intercourse: Implications for Rectal Microbicides. AIDS Behav. 2008, 12, 860-866. [CrossRef]

20. Ucciferri, C.; Tamburro, M.; Falasca, K.; Sammarco, M.L.; Ripabelli, G.; Vecchiet, J. Prevalence of Anal, Oral, Penile and Urethral Human Papillomavirus in HIV Infected and HIV Uninfected Men Who Have Sex with Men. J. Med. Virol. 2018, 90, 358-366. [CrossRef]

21. Hart, T.L.; Coon, D.W.; Kowalkowski, M.A.; Zhang, K.; Hersom, J.I.; Goltz, H.H.; Wittmann, D.A.; Latini, D.M. Changes in sexual roles and quality of life for gay men after prostate cancer: Challenges for sexual health providers. J. Sex. Med. 2014, 11, 2308-2317. [CrossRef]

22. Johns, M.M.; Pingel, E.; Eisenberg, A.; Santana, M.L.; Bauermeister, J. Butch tops and femme bottoms? Sexual positioning, sexual decision making, and gender roles among young gay men. Am. J. Men's Health 2012, 6, 505-518. [CrossRef] [PubMed]

23. Wei, C.; Raymond, H.F. Preference for and Maintenance of Anal Sex Roles among Men Who Have Sex with Men: Sociodemographic and Behavioral Correlates. Arch. Sex. Behav. 2011, 40, 829-834. [CrossRef] [PubMed]

24. Nugraheni, P.; Tobing, H.; Kusumadewi, I.; Siste, K.; Putri, N. Is it important to assess mental health problem in MSM? Asian J. Psychiatry 2018, 101584. [CrossRef] [PubMed] 
25. Bostwick, W.B.; Boyd, C.J.; Hughes, T.L.; McCabe, S.E. Dimensions of Sexual Orientation and the Prevalence of Mood and Anxiety Disorders in the United States. Am. J. Public Health 2010, 100, 468-475. [CrossRef] [PubMed]

26. Parker, R.D.; Lõhmus, L.; Valk, A.; Mangine, C.; Rüütel, K. Outcomes associated with anxiety and depression among men who have sex with men in Estonia. J. Affect. Disord. 2015, 183, 205-209. [CrossRef]

27. Fendrich, M.; Avci, O.; Johnson, T.P.; Mackesy-Amiti, M.E. Depression, Substance Use and Hiv Risk in a Probability Sample of Men Who Have Sex with Men. Addict. Behav. 2013, 38, 1715-1718. [CrossRef]

28. Hanrahan, N.P.; Wu, E.; Kelly, D.; Aiken, L.H.; Blank, M.B. Randomized Clinical Trial of the Effectiveness of a Home-Based Advanced Practice Psychiatric Nurse Intervention: Outcomes for Individuals with Serious Mental Illness and HIV. Nurs. Res. Pract. 2011, 2011, 840248. [CrossRef]

29. Pappin, M.; Wouters, E.; Booysen, F.L. Anxiety and depression amongst patients enrolled in a public sector antiretroviral treatment programme in South Africa: A cross-sectional study. BMC Public Health 2012, 12, 244. [CrossRef]

30. Chen, F.; Ding, F.; Lin, X.; Wang, X.; He, H.; Huang, W.; Dong, Y.; Yang, Q.; Zhang, H. Prevalence Rates of Depression and Anxiety in HIV-Infected Men Who Have Sex with Men. Chin. Ment. Health J. 2015, 29, 251-257. (In Chinese)

31. Szymańska-Kotwica, B.; Cholewińska, G.; Cielniak, I.; Firlag-Burkacka, E.; Łojek, E.; Horban, A.; Bornstein, R. Prevalence of depression and anxiety symptoms in HIV-positive men who have sex with men treated with effective cART. J. Psychosom. Res. 2018, 109, 138. [CrossRef]

32. Tran, B.X.; Ho, R.C.M.; Ho, C.S.H.; Latkin, C.A.; Phan, H.T.; Ha, G.H.; Vu, G.T.; Ying, J.; Zhang, M.W.B. Depression among Patients with HIV/AIDS: Research Development and Effective Interventions (GAPRESEARCH). Int. J. Environ. Res. Public Health 2019, 16, 1772. [CrossRef]

33. Clark, J.; Salvatierra, J.; Segura, E.R.; Salazar, X.; Konda, K.; Perez-Brumer, A.; Hall, E.; Klausner, J.; Caceres, C.; Coates, T. Moderno love: Sexual role-based identities and HIV/STI prevention among men who have sex with men in Lima, Peru. AIDS Behav. 2013, 17, 1313-1328. [CrossRef] [PubMed]

34. Zung, W.W. A Rating Instrument for Anxiety Disorders. Psychosomatics 1971, 12, 371-379. [CrossRef]

35. Koblin, B.; Chesney, M.; Coates, T. Effects of a behavioural intervention to reduce acquisition of HIV infection among men who have sex with men: The EXPLORE randomised controlled study. Lancet 2004, 364, 41-50. [CrossRef]

36. Ying, M.; Zhang, Y.; Song, G. Research on Epidemic Model of Emergency Events Risk Perception in Food Industry. Sci. Res. Manag. 2013, 34, 123-130. (In Chinese)

37. Bradley, H.; Tsui, A.; Hindin, M.; Kidanu, A.; Gillespie, D. Developing scales to measure perceived HIV risk and vulnerability among Ethiopian women testing for HIV. AIDS Care 2011, 23, 1043-1052. [CrossRef] [PubMed]

38. Yun, K.; Zhang, J.; Chu, Z.; Hu, Q.; Geng, W.; Jiang, Y.; Wang, N.; Xu, J. Association between Psychological Disorders, Depression and the Cd4 Level of HIV Negative MSM in Shenyang. Chin. J. AIDS STD 2018, 24, 1115-1118. (In Chinese)

39. Hylton, E.; Wirtz, A.L.; Zelaya, C.E.; Latkin, C.; Peryshkina, A.; Mogilnyi, V.; Dzhigun, P.; Kostetskaya, I.; Galai, N.; Beyrer, C. Sexual Identity, Stigma, and Depression: The Role of the "Anti-gay Propaganda Law" in Mental Health among Men Who Have Sex with Men in Moscow, Russia. J. Hered. 2017, 94, 319-329. [CrossRef]

40. Stoloff, K.; Joska, J.A.; Feast, D.; De Swardt, G.; Hugo, J.; Struthers, H.; McIntyre, J.; Rebe, K. A Description of Common Mental Disorders in Men Who Have Sex with Men (MSM) Referred for Assessment and Intervention at an MSM Clinic in Cape Town, South Africa. AIDS Behav. 2013, 17 (Suppl. 1), S77-S81. [CrossRef]

41. De Santis, J.P.; Colin, J.M.; Vasquez, E.P.; McCain, G.C. The Relationship of Depressive Symptoms, Self-Esteem, and Sexual Behaviors in a Predominantly Hispanic Sample of Men Who Have Sex with Men. Am. J. Men's Health 2008, 2, 314-321. [CrossRef]

42. Chen, C.; Wang, M.; Wang, T.; Li, L.; Lai, X.; Sun, Y.; Chen, J. Analysis of the Sex Roles in the Relationship with the HIV/Syphilis Infection and the Related Behaviors among Men Who Have Sex with Men in Zhongshan City. J. Public Health Prev. Med. 2016, 27, 53-56. (In Chinese) 
43. Jorm, A.F.; Korten, A.E.; Rodgers, B.; Jacomb, P.A.; Christensen, H. Sexual orientation and mental health: Results from a community survey of young and middle-Aged adults. Br. J. Psychiatry 2002, 180, 423-427. [CrossRef] [PubMed]

44. Secor, A.M.; Wahome, E.; Micheni, M.; Rao, D.; Simoni, J.M.; Sanders, E.J.; Graham, S.M. Depression, substance abuse and stigma among men who have sex with men in coastal Kenya. AIDS 2015, 29, S251-S259. [CrossRef] [PubMed]

45. Kramer, S.C.; Schmidt, A.J.; Berg, R.C.; Furegato, M.; Hospers, H.; Folch, C.; Marcus, U.; Network, E. Factors associated with unprotected anal sex with multiple non-steady partners in the past 12 months: Results from the European Men-Who-Have-Sex-with-Men Internet Survey (EMIS 2010). BMC Public Health 2016, 16, 47. [CrossRef] [PubMed]

46. Peng, X.; Li, L.; Qi, J.; Chen, M. Status of Anxiety and Depression and Related Influence Factors among Men Who Have Sex with Man in Shenzhen. Chin. J. AIDS STD 2017, 23, 630-633. (In Chinese)

47. Chen, C.; Wang, M.; Wang, T.; Li, L.; Lai, X.; Sun, Y.; Chen, J. Impact of the Sexual Roles in the Relationship with the HIV Infection and the Related Behaviors among Men Who Have Sex with Men, Zhongshan City. Mod. Prev. Med. 2016, 43, 99-102. (In Chinese)

48. Zhang, J.; Li, Q.; Han, D.; Wang, X.; Wu, C.; He, Q.; Liu, S.; Nuan, R. The Sexual Role Status and Its Relationship with Condom Use and HIV-Testing in Men Who Have Sex with Men. Chin. J. Dis. Control Prev. 2013, 17, 400-403. (In Chinese)

49. Alvy, L.M.; McKirnan, D.J.; Mansergh, G.; Koblin, B.; Colfax, G.N.; Flores, S.A.; Hudson, S.; Project MIX Study Group. Depression is Associated with Sexual Risk among Men Who Have Sex with Men, but is Mediated by Cognitive Escape and Self-Efficacy. AIDS Behav. 2011, 15, 1171-1179. [CrossRef]

50. Gilman, S.E.; Cochran, S.D.; Mays, V.M.; Hughes, M.; Ostrow, D.; Kessler, R.C. Risk of psychiatric disorders among individuals reporting same-sex sexual partners in the National Comorbidity Survey. Am. J. Public Health 2001, 91, 933-939.

51. Tao, J.; Qian, H.-Z.; Kipp, A.M.; Ruan, Y.; Shepherd, B.E.; Amico, K.R.; Shao, Y.; Lu, H.; Vermund, S.H. Effects of depression and anxiety on antiretroviral therapy adherence among newly diagnosed HIV-infected Chinese MSM. AIDS 2017, 31, 401-406. 